\title{
The utility of the Personality Clinical Form Indicators of Response Distortion: Receiver Operating Characteristic Analysis
}

\author{
Florin A. Sava ${ }^{1 *}$, Camelia Dindelegan ${ }^{2} \&$ Nastasia Sălăgean ${ }^{1}$ \\ ${ }^{1}$ Department of Psychology, West University of Timişoara, Romania. \\ ${ }^{2}$ Department of Psychology, University of Oradea, Romania.
}

Received 20.12.2019; Received revised 22.06.2020; Accepted 28.06.2020 Available online 30.06 .2020

\begin{abstract}
A Receiver Operating Characteristic Analysis (ROC Analysis) was conducted to assess the efficiency of six validity scales included in the Personality Clinical Form (PCF) to detect responses distortion. Undergraduate students were randomly assigned to simulate malingering, simulate defensiveness or complete PCF under standard instructions (no faking). Fake-good participants scored significantly higher than standard participants on all underreporting scales. The difference observed was even higher when the comparison was made between the fake-good and the fake-bad participants. Likewise, a reverse trend was observed for the overreporting scales. Participants in the fake-bad condition scored the highest, and the participants in the fake-good condition scored the lowest on all overreporting scales. Large effect sizes were found in most cases. The responses resulted from the malingering condition were also compared with those obtained from psychiatric inpatients. The responses resulted from the defensiveness group were also compared with responses obtained from employees in a high-stake assessment condition. The area under the ROC curve (AUC) provided an index of discriminative power. The validity scales discriminate better between the normal and the fake conditions than between malingerers and psychiatric inpatients, but most AUC values were within good or excellent range. Cut-off scores and their corresponding sensitivity and specificity were presented for each validity scale based on this explorative endeavour.
\end{abstract}

Keywords: sensitivity, specificity, personality assessment, personality disorders, malingering, PCF.

Address of correspondence: Florin A. Sava, Department of Psychology, West University of Timișoara, 4 Vasile Pârvan Blvd., 300223 Timișoara, Romania.

E-mail: florin.sava@e-uvt.ro

Data availability statement: Data that support this study's findings are openly available in the Open Science Framework at doi: 10.17605/OSF.IO/U5Q98.

\section{Introduction}

In any personality assessment that relies on self-report instruments, a critical question that always arises is whether we can rely on these responses or whether participants provided a distorted response. The distortion could either be directed towards exaggerating (overreporting, fake bad) or towards minimizing the pathology (underreporting, fake good). For instance, applicants for a job, divorcing parents who undergo a custody evaluation or prisoners who require benefits such as a transfer to an institution with better facilities, will often try to portray themselves in a positive picture, underreporting their personality issues. On the contrary, patients under severe stress (genuine sufferers) and malingering participants who deliberately exaggerate their symptoms to avoid some legal actions or to gain financial compensation through litigation tend to portray themselves in a distorted negative picture, by overreporting their personality issues. Likewise, personality traits are strongly related to affect (Kritzler, Krasko \& Luhmann, 2020), so that stressful life events could alter self-reports (Chiracu, 2019).

The most well-known personality and psychopathology instruments in clinical assessment have addressed these concerns by incorporating validity scales to identify 
abnormal response patterns. It is valid for the MMPI-2 (Butcher, Dahlstrom, Graham, Tellegen, \& Kaemmer, 1989; see also Baer, Wetter, Nichols, Greene, \& Berry, 1995; Nicholson et al., 1997), for the MCMI-IV (Millon, Grossman, \& Millon, 2015; see also Daubert and Metzler, 2000; Schoenberg, Dorr \& Morgan, 2003), for the PAI (Morey, 2007; see also Hawes \& Boccaccini, 2009). Another well-known personality inventory, the PID-5 (Krueger, Derringer, Markon, Watson, \& Skodol, 2013), based on the alternative model for personality disorders in the Diagnostic and Statistical Manual of Mental Disorders (5 ${ }^{\text {th }}$ ed., DSM-5; American Psychiatric Association, 2013) does not include validity scales. More recently, some authors suggested adding an overreporting scale (Selbom, Dhillon, \& Bagby, 2018), of an inconsistency scale (Bagby and Selbom, 2018), and two impression management scales (Williams, Rogers, Sharf, \& Ross, 2019) to allow detecting the patterns of responding. However, up to this moment, these scales were not officially included in the structure of the PID-5.

The Personality Clinical Form (PCF) is a 200-item newly developed instrument (Sava, in press) designed to assess personality disorders (PDs) accounted by the DSM5. The PCF overcomes several PID-5 limitations. Firstly, it includes six validity scales to detect three patterns of responding: overreporting, underreporting, and random answers. Secondly, unlike the PID-5, which was limited to assessing the personality trait model used in Criterion B of the alternative model of personality disorders (e.g., the 25 "lower-order" trait scales and the five personality domains), the PCF is more comprehensive. It covers both the ten well-known personality disorders (e.g., histrionic personality disorder) and the alternative model of personality disorders. In the latter case, it assesses criterion A (levels of dysfunctionality in self and interpersonal relations) and criterion $\mathrm{B}$ that was targeted by PID-5 (personality traits and domains). Thirdly, unlike the PID-5, which uses rational cut-off scores and disregards differences in item difficulties across the scales, the PCF uses empirically derived cut-off scores through norms and considers the differences in item difficulties across scales by providing normalized $\mathrm{T}$ scores for the interpretation of the results. Last but not least, whereas the PID-5 uses a 4point Likert scale to collect answers, the PCF uses a dichotomous format (True/False) for answers, this format being more appropriate for people with little formal education and less susceptible of cultural bias (Flaskerud, 2012).

The study's purpose was to evaluate how well each of the six validity scales was able to detect distorted responses (underreporting, overreporting, random). Before proceeding to the method section, we will summarize the six validity scales included in the PCF. Table 1 also contains the logic used in the construction of the scales and references to analogous scales found in other well-known instruments.

\section{Method}

\section{Participants}

We employed a sophisticated strategy for data collection. The main group of participants consisted of 145 undergraduate psychology students. Of these, 83 students completed the PCF test in a standard condition, 30 students completed the PCF test under fake-good instructional sets, and 32 students completed the PCF test under the fake-bad instructional sets.
Data collected from two reference groups were also included in this study. The first group consisted of 68 employees who completed the test under a high-stake context during a compulsory psychological assessment session, as a part of a work safety procedure that could affect their employment status. The second group consisted of 34 general psychiatric inpatients of various diagnostics. Both reference groups had undertaken the PCF under standard instructions. Besides, 100 profiles of PCF were randomly generated using the runiform command from the Stata software (StataCorp., 2017), and this group was also used as a reference group for the detection of random patterns of response.

No statistical power analysis was conducted to determine the sample size, and all participants selected in this study were recruited non-probabilistically as a convenience sample.

\section{Measures}

The PCF (Sava, in press) is a 200 -item instrument for assessing personality disorders based on the DSM-5 conceptualization. We focused on the six validity scales: inconsistency (IC), infrequent responses (IF), distress (DT), disclosure amplitude (DA), desirability (DS), and defensiveness (DF).

The MMPI-2 S scale (Butcher \& Hahn, 1995) is a 50 item scale used to detect underreporting. Individuals who obtain high scores at this scale proclaim extreme virtues and the absence of psychopathology. We added this scale for comparison purposes.

\section{Procedure}

Students were recruited to participate in a study examining the ability of the PCF test to detect participants who attempted to fake bad or fake good their responses at the test. Students completed a paper-and-pencil version of the PCF test in a group context. Clusters of students were randomly assigned to the experimental conditions. Several scenarios were provided for the fake-good and fake-bad condition to increase the students' motivation to perform the dissimulation. For instance, for the malingering condition, they could choose from: (a) an insanity scenario to avoid criminal conviction; (b) an injury scenario to receive compensations through litigation; (c) a workrelated scenario to receive disability benefits or reduce their working hours. Likewise, for the defensiveness condition, they could choose from several scenarios: (a) gaining custody of a child in a divorce setting; (b) securing release from a psychiatric institution; (c) applying for a job position. The instructions in both faking conditions stressed the importance of responding believably not to be identified as either exaggerating or minimizing symptoms. We also provided the participants the opportunity to win 100 lei in a lottery if the validity scales detect them neither as malingerers nor as defenders to stimulate their dissimulation effort. Likewise, the students in the standard condition were told they would receive feedback on their results to promote honesty in their responses. All three groups of students received extra-credit for their participation in this study. It is also important to note that all three groups of students were aware of various forms of the validity scales, as they were enrolled in a Personality Tests course during the time of testing. They were also familiar with various mental disorders symptoms as they were also enrolled in a Psychopathology course in parallel. However, no specific test coaching on dissimulating at the PCF was provided. 
The employees also had taken the PCF in a groupsetting using the paper-and-pencil format of the test. They completed the test together with another measure not included in the study as a part of a compulsory psychological examination by law. They were told that the PCF was used for research purposes only after completing the tests to maintain the high-stake assessment context. Last but not least, the PCF was also completed in an individual setting by a group of general psychiatric inpatients with various diagnostics. These participants completed the PCF voluntarily, anonymously, being informed in advance that their effort is made for research purposes. Patients experiencing an acute episode of psychotic disorder were not eligible to be included in the study.

\section{Results}

All analyses were conducted using Stata 15 (StataCorp, 2017). The first step in the analysis required the investigation of between-groups differences between the three experimental conditions (standard vs. fake good vs. fake bad). The main results are presented in Table 2, which also contains the resulted statistics from the randomly generated data.

Differences between the three experimental groups (fake-good vs. standard vs. fake-bad) were examined with one-way ANOVA. Because of the large number of statistical inference tests conducted, we used a conservative p-value of .001 for the statistical significance. All differences were statistically significant in the expected direction. For instance, participants undertaking the PCF test scored highest on the DS and DF scales in a fake good condition. They also scored high on the $\mathrm{S}$ scale from MMPI-2. Likewise, participants in the fake bad condition scored highest on the IF, DT, and DA scales, and participants in the random condition scored highest on the IC scale. However, three of the indicators - DF, DT, and DA seem to play a bidirectional role. For instance, high scores of DF, along with low levels of DT and DA, indicate underreporting (fake-good tendencies), whereas a reversed pattern indicates overreporting (fake-bad tendencies). Figure 1 provides the average responses for the six validity scales using normalized $\mathrm{T}$ scores as a unit of measurement.

Table 1. Detection strategies for distorted responses in the PCF

\begin{tabular}{|c|c|c|c|}
\hline $\begin{array}{l}\text { Primary } \\
\text { purpose }\end{array}$ & Scale name & Strategy description & Analog scales \\
\hline $\begin{array}{l}\text { Over- } \\
\text { reporting or } \\
\text { random } \\
\text { responses }\end{array}$ & Infrequency (IF) & $\begin{array}{l}\text { It focuses on the endorsement of rare and quasi-rare symptoms. } \\
\text { Malingerers tend to overreport such symptoms to a higher } \\
\text { degree than genuinely distressed participants. Likewise, a } \\
\text { random pattern of responses leads to a high level of IF. }\end{array}$ & $\begin{array}{l}\text { F, Fp scales from } \\
\text { MMPI-2 }\end{array}$ \\
\hline $\begin{array}{l}\text { Over- } \\
\text { reporting }\end{array}$ & Distress (DT) & $\begin{array}{l}\text { It focuses on spotting participants with a high level of emotional } \\
\text { distress. These participants have a high probability of } \\
\text { overreporting their symptoms. The scale is not intended to } \\
\text { discriminate between genuine distress and malingering, but it is } \\
\text { useful for detecting distorted responses (overreporting) that } \\
\text { requires the calibration of scores. }\end{array}$ & $\begin{array}{l}\text { CD scale from } \\
\text { OMNI-IV }\end{array}$ \\
\hline $\begin{array}{l}\text { Over- } \\
\text { reporting* }\end{array}$ & $\begin{array}{l}\text { Disclosure amplitude } \\
\text { (DA) }\end{array}$ & $\begin{array}{l}\text { It indiscriminately focuses on symptom endorsement. } \\
\text { Malingerers tend to endorse more symptoms in comparison with } \\
\text { genuine sufferers. }\end{array}$ & $\begin{array}{l}X \text { scale from the } \\
\text { MCMI-IV }\end{array}$ \\
\hline $\begin{array}{l}\text { Under- } \\
\text { reporting }\end{array}$ & Defensiveness (DF) & $\begin{array}{l}\text { The scale ranges on a continuum from a self-critical attitude and } \\
\text { over-declaration of symptoms (low DF) to a tendency to be } \\
\text { defensive and to portray oneself in favorable terms, being } \\
\text { reluctant to admit any problem (high DF). Defensive } \\
\text { participants score very high on this scale. }\end{array}$ & $\begin{array}{l}\mathrm{K} \text { and } \mathrm{S} \text { scales } \\
\text { from MMPI- } 2\end{array}$ \\
\hline $\begin{array}{l}\text { Under- } \\
\text { reporting }\end{array}$ & Desirability (DS) & $\begin{array}{l}\text { Focuses on endorsing unlikely virtues. It is traditionally seen as } \\
\text { a scale that detects intentional effort to under-report, but it could } \\
\text { also result from an unrealistic positive self-image. Defensive } \\
\text { participants score very high on this scale. }\end{array}$ & $\begin{array}{lcc}\text { L } & \text { scale } & \text { from } \\
\text { MMPI-2** } & \end{array}$ \\
\hline $\begin{array}{l}\text { Random } \\
\text { responses }\end{array}$ & Inconsistency (IC) & $\begin{array}{l}\text { It focuses on detecting cases where participants respond in a } \\
\text { contradictory manner in selected pairs of items. The higher the } \\
\text { score, the more likely the probability of a person to respond } \\
\text { inconsistently (randomly, not carefully). }\end{array}$ & $\begin{array}{l}\text { VRIN scale from } \\
\text { MMPI-2 }\end{array}$ \\
\hline
\end{tabular}

* DA scale could also be relevant for detecting underreporting, defensive individuals reporting very few symptoms compared with the non-clinical population.

** Unlike the L scale from MMPI-2, where items are phrased to enhance denial (absence of moral imperfection), in the DS scale from PCF items are phrased to enhance desirability (the presence of moral virtues). 
Table 2. Relevant descriptive and inferential statistics for the validity scales of PCF

\begin{tabular}{|c|c|c|c|c|c|c|c|c|c|c|c|c|}
\hline \multirow[b]{2}{*}{ Scale } & \multicolumn{2}{|c|}{ Fake Good } & \multicolumn{2}{|c|}{ Standard } & \multicolumn{2}{|c|}{ Fake Bad } & \multirow[b]{2}{*}{$\mathrm{F}$} & \multicolumn{2}{|c|}{ Random } & \multicolumn{3}{|c|}{ Effect sizes } \\
\hline & $\mathrm{M}$ & SD & $\mathrm{M}$ & SD & $\mathrm{M}$ & SD & & $\mathrm{M}$ & SD & $\mathrm{d}_{\mathrm{FG}}$ & $\mathrm{d}_{\mathrm{FB}}$ & $\mathrm{d}_{\mathrm{R}}$ \\
\hline DS & 9.1 & 3.4 & 6.1 & 3.2 & 4.7 & 3.2 & $14.6^{*}$ & 5.9 & 1.6 & .90 & 0.41 & 0.06 \\
\hline DF & 19.4 & 3.6 & 13.1 & 4.9 & 4.9 & 4.0 & $81.1^{*}$ & 11.6 & 2.1 & 1.46 & 1.80 & 0.37 \\
\hline IF & 1.0 & 1.3 & 2.3 & 2.0 & 8.4 & 2.7 & $13.5^{*}$ & 7.0 & 1.9 & 0.73 & 2.53 & 2.36 \\
\hline DT & 0.9 & 1.3 & 3.3 & 2.8 & 10.2 & 2.2 & $126.9^{*}$ & 6.0 & 2.0 & 1.10 & 2.70 & 1.08 \\
\hline DA & 27.1 & 14.2 & 46.2 & 20.7 & 94.4 & 19.9 & $104.7 *$ & 77.6 & 6.1 & 1.07 & 2.37 & 2.05 \\
\hline IC & 3.3 & 1.5 & 4.1 & 1.9 & 4.4 & 1.9 & 2.7 & 10.6 & 3.3 & 0.45 & 0.13 & 2.40 \\
\hline $\mathrm{S}_{\mathrm{MMPI}}$ & 35.1 & 8.6 & 23.4 & 7.9 & 14.6 & 5.7 & $57.4 *$ & - & - & 1.41 & 1.28 & - \\
\hline
\end{tabular}

Legend: DS (Desirability), DF (Defensiveness), IF (Infrequent responses), DT (Distress), DA (Disclosure amplitude), IC (Inconsistency); $\mathrm{d}_{\mathrm{FG}}$ is the Cohen's $\mathrm{d}$ effect size indicator for the difference between Fake Good and Standard conditions; $\mathrm{d}_{\mathrm{FB}}$ is the Cohen's d effect size indicator for the difference between Fake Bad and Standard conditions; $d_{R}$ is the Cohen's d effect size indicator for the difference between Random and Standard conditions.

$*$ for $\mathrm{p} s<.001$; bold fonts were used to emphasize our hypotheses based on the indicators' rationale.

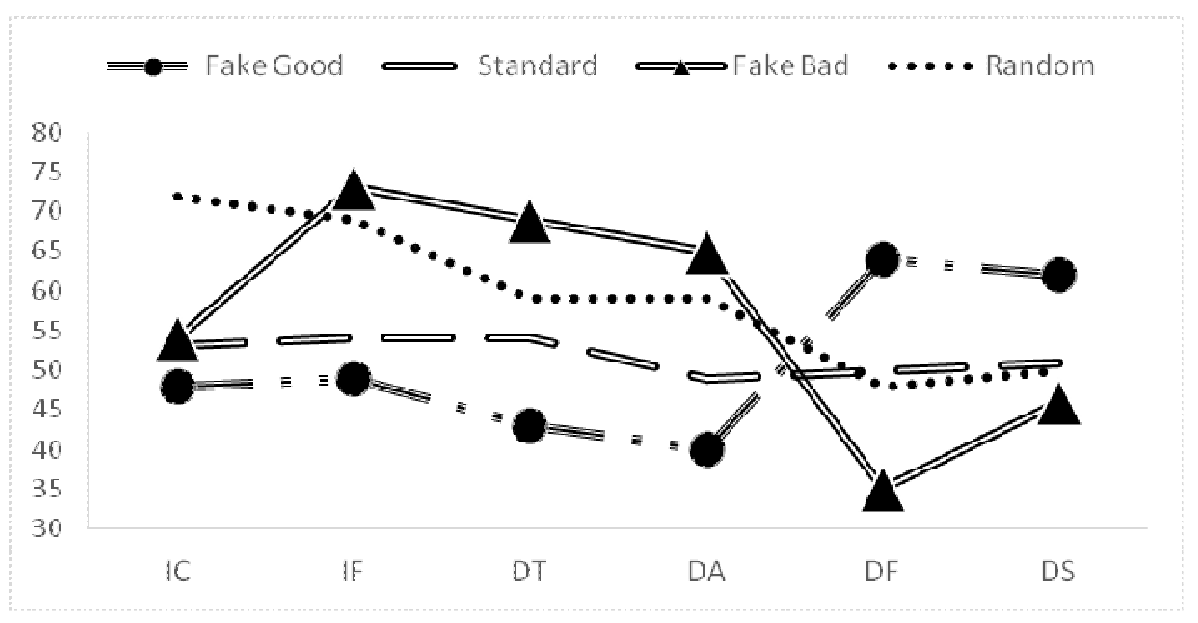

Figure 1. Patterns of responses on validity scales under various experimental conditions

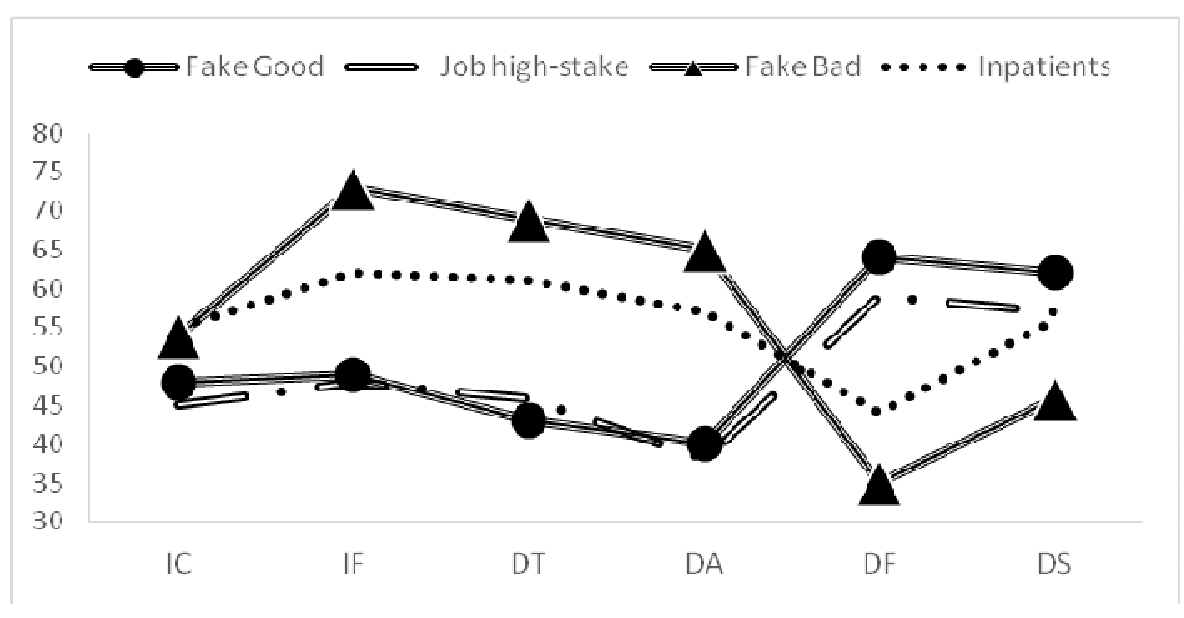

Figure 2. Patterns of responses on validity scales in various assessment contexts 
Table 3. Classification accuracy. Discriminating between target conditions (fake good/fake bad/random) and relevant comparison groups (standard instruction or psychiatric inpatients)

\begin{tabular}{lllllllll}
\hline Population & Scale & AUC & Cut-off & T & Hit Rate & Sensitivity & Specificity & LR+ \\
\hline Fake good vs. standard & DS & $.819(.055)$ & $>=10$ & 66 & $79.75 \%$ & $60.00 \%$ & $91.84 \%$ & 7.3 \\
& DF & $.866(.041)$ & $>=20$ & 65 & $81.01 \%$ & $63.33 \%$ & $91.84 \%$ & 7.7 \\
& S & $.837(.048)$ & $>=34$ & - & $79.75 \%$ & $60.00 \%$ & $91.84 \%$ & 7.3 \\
\hline Fake bad vs. standard & IF & $.944(.023)$ & $>=7$ & 69 & $85.19 \%$ & $71.88 \%$ & $93.88 \%$ & 11.7 \\
& DT & $.946(.024)$ & $>=9$ & 65 & $90.12 \%$ & $81.25 \%$ & $95.92 \%$ & 19.9 \\
& DA & $.941(.028)$ & $>=81$ & 60 & $88.90 \%$ & $81.25 \%$ & $93.88 \%$ & 13.3 \\
\hline Random vs. standard & IF & $.939(.018)$ & $>=6$ & 67 & $83.61 \%$ & $77.00 \%$ & $91.57 \%$ & 9.1 \\
& IC & $.950(.014)$ & $>=8$ & 66 & $88.52 \%$ & $83.00 \%$ & $95.12 \%$ & 17.2 \\
\hline Fake bad vs. inpatients & DF & $.782(.056)$ & $<=2$ & 23 & $66.67 \%$ & $34.38 \%$ & $97.06 \%$ & 11.7 \\
& IF & $.813(.052)$ & $>=11$ & 77 & $65.15 \%$ & $31.25 \%$ & $97.06 \%$ & 10.6 \\
& DT & $.759(.058)$ & - & - & - & - & - & - \\
& DA & $.762(.059)$ & - & - & - & - & - & - \\
\hline
\end{tabular}

Notice: cut-off values were selected (a) to ensure very high specificity of at least .90, (b) high level of hit rates (correct classifications), and (c) high values of positive likelihood ratios (typically higher than 10.0, if possible).
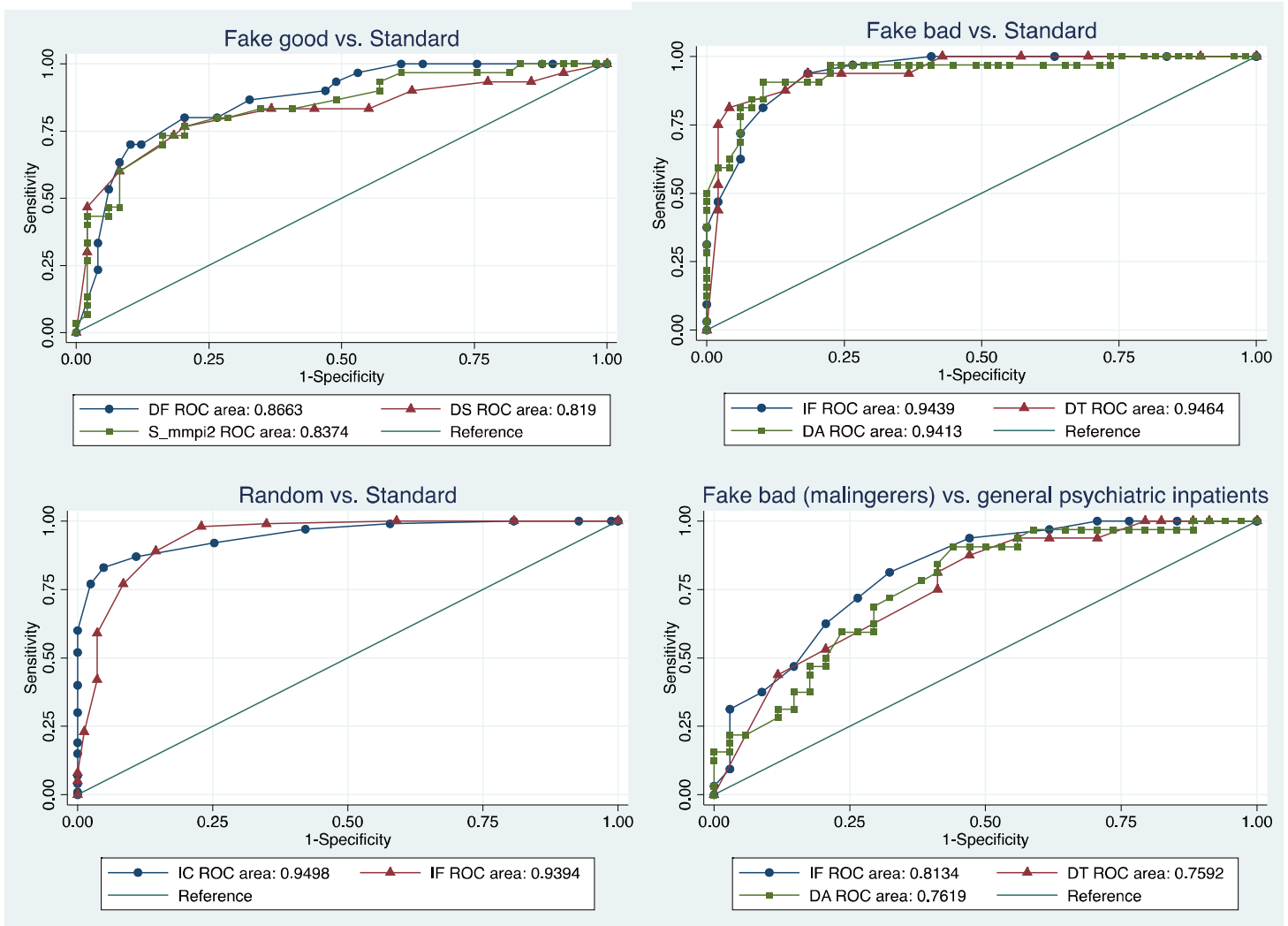

Figure 3. Receiver operating characteristic curve for the main comparisons

We also examined whether the validity scales provide the same pattern of response in testing scenarios that trigger what might be called positive malingering (e.g., fake good experimental condition vs. employees in a highstake assessment context). Overall, the patterns of responses replicated the expected direction, finding similarities between the fake good and high-stake context conditions. However, we did find a significant statistical difference for the DS scale $(\mathrm{d}=0.42)$ and a marginal statistical difference for the DF scale $(d=0.33)$, with slightly higher levels of distortions in responses in the fake good condition.
Likewise, we examined whether the validity scales provide a different response pattern between the two overreporting contexts that differed in terms of malingering probability (high for the fake bad experimental condition vs. low for the psychiatric inpatients). All validity scales, excepting for the IC scales, revealed significant differences between these two conditions, with an increased pattern of overreporting found in the high malingering context. Thus, we found significant statistical differences for the IF scale $(\mathrm{d}=1.28)$, for the DT scale $(\mathrm{d}=1.03)$, for the DA scale $(\mathrm{d}$ $=1.01)$, for the DS scale $(\mathrm{d}=0.88)$, and for the DF scale $(\mathrm{d}$ $=1.10$ ). Figure 2 provides the pattern of response for the 
six validity scales using normalized $\mathrm{T}$ scores as a unit of measurement in these four conditions.

\section{ROC analyses}

We employed a ROC analysis to provide additional insights into the validity scales utility. The ROC analysis is used in classification problems, for instance, to test whether a specific indicator is capable of distinguishing between two specific situations (e.g., a fake bad profile vs. a valid profile). The ROC quantifies a test's accuracy by estimating the area under the curve (AUC) value (Youngstrom, 2014). Among several advantages of using such an analysis, we would like to emphasize that the AUC value can be interpreted straightforwardly. It is the probability that a random participant selected from the target condition (e.g., fake-good) scores higher on that scale than a randomly chosen individual from the reference group (e.g., standard instructions). The AUC is also very helpful in selecting the cut-off score and allows for computing sensitivity and specificity for each possible cutoff score. A rough guide to interpreting the AUC values (Sava, 2013) suggests that values over .90 are excellent, values of $.80-.90$ values are good and values of $.70-.80$ are fair/acceptable.

\section{Performance of the underreporting scales}

We observed that the AUC values to detect fake good profiles were typically smaller than those used to detect fake bad profiles. Nevertheless, it was revealed good discrimination of normal vs. fake-good participants, obtaining AUC values higher than .80. Likewise, both DF and DS validity scales from PCF had similar performance to detect underreporting as the well-known MMPI-2 Superlative self-presentation scale (S). There were no statistical differences among the three AUC values $-\chi^{2}(2)$ $=1.19, \mathrm{p}=.552$.

\section{Performance of the overreporting scales}

The AUC values for all three scales designed to detect overreporting - IF, DT and DA were very high (around .95 ), revealing excellent discrimination of normal and fakebad participants, and no statistical differences among the three AUC values - $\chi^{2}(2)=0.03, p=.984$. However, overreporting does not equal malingering because it could also be originated from genuine emotional distress, as in the case of psychiatric inpatients. The validity scales had a fair to good ability to discriminate between psychiatric inpatients and malingerers (an AUC value below .80 in most cases). Because of this reason, we retain only the IF and the DF scales to discriminate between genuine sufferers and malingerers. However, as noticed from Figure 2, malingerers manifest extreme overreporting on all scales except for IC. The difference in response between malingerers and genuine sufferers is typically large (Cohen's $\mathrm{d} s>1.0)$.

\section{Performance of random responses scales}

The AUC values suggest excellent discrimination abilities between standard profiles and profiles generated based on random answers (e.g., in practical settings because of shallow motivation). The AUC values were close to .95 for both IC and IF scales. There were no statistical differences among the two AUC values $-\chi^{2}(1)=$ $0.24, \mathrm{p}=.621$. As expected, profiles containing random responses contain more infrequent responses and more inconsistent responses (see Figure 3).

\section{Discussion}

The present study aimed to evaluate how well each of the six validity scales of PCF can detect distorted responses. The results suggest that the underreporting indicators, DF and DS, are useful to discriminate between regular and fake good profiles. Likewise, overreporting indicators such as DA, DT, and IF were very performant in discriminating between regular and fake bad profiles. The IC and the IF scales were also highly performant in discriminating between normal and randomly generated responses.

We have also noticed a strategy to improve the detection of both under- and overreporting profiles. Several indicators play a bidirectional role (e.g., a very low score on DF indicates overreporting, while a very high score on DF indicates underreporting). As can be seen from figure 1 , the average profile of overreporting, and the average profile of underreporting seems to mirror each other. Thus, the more pronounced the underreporting pattern (very high levels of DF and DS and very low levels of IF, DT, DA), the more likely it is to encounter an intentional fake good scenario. Likewise, a reverse pattern of response (very low scores for DF and DS, and very high scores for IF, DT, and DA) will signal an intentional overreporting (malingering scenario). For instance, employing a composite Fake Good Index (FGI $=$ DF + DS - DT) increases the AUC to .896 and the hit rate to $83.54 \%$, with a sensitivity of $63.33 \%$ and a specificity of $95.92 \%$ for a cut-off score $\geq 28$ for this mixed indicator.

The random profile could be detected by observing high levels of IC and IF. However, we should notice that a high IF is also found in the case of overreporting profiles. To resolve this ambiguity, we should also look at the pattern of the associated scales with high IF. A high score on the IF scale it is usually associated with high scores on DT and very low scores on DF in an overreporting profile. Instead, in a random response profile, a high score on IF is usually associated with IC's high values.

We also aimed to look at how good the PCF scales are to differentiate between two types of overreporting, namely genuine distress, which is typically found in psychiatric inpatients and malingering, which is an intentionally overreport of symptoms to gain benefits or avoid penalties. We obtained a fair to good performance to discriminate between genuine distress and malingerers (AUC values around .80 and large effect sizes for five out of the six validity scales). We found that malingerers tend to exaggerate their suffering, In line with the existing literature (e.g., Rogers, Sewell, Martin, and Vitacco, 2003). This pattern of response could be best detected in the case of PCF by very high scores of IF and very low scores on DF. Luckily, besides the statistical performance of the validity scales, there is other relevant information that should be taken into account by a clinician to differentiate between malingerers and distressed patients showing a "cry for help" profile (see, for instance, Bass and Halligan, 2014). The most obvious difference is that there should be a significant reason (stake) for malingering. Patients coming for psychotherapy because of distress tend to have a low motivation to malinger. Instead, persons who have been tested in a medicolegal setting and whose responses have legal/financial implications are more likely to malinger. In the presence of a reason to malinger, other discriminating clues could be used. For instance, marked or unexpected differences between the patient self-reporting claims and the clinician observations, or the third-source 
information raise doubts about the credibility of the selfreport. Inconsistencies between test results and the patient life history, inconsistencies in the stories revealed by the patient during the interview, or concerning the present condition should also be confronted. Usually, relying on other-informant sources is typical if malingering is suspected.

Several limitations of the current investigation should be taken into account. First, we should also note that the undergraduate psychology students from this study were both familiar with the concept of validity scales and psychopathology concepts, thus sharing such characteristics with coached participants (e.g., Baer, Wetter, \& Berry, 1995). Such a sample could reduce the discriminative power of the validity scales to differentiate between malingerers and genuine sufferers. Further research should investigate more directly the effect of coaching on the power of discriminating between target and reference conditions.

Likewise, there was no statistical power analysis conducted before the collection of data. The small sample size affects the robustness of the statistical estimators (e.g., the AUC values) and provides rather large confidence intervals. The use of college students in the three experimental conditions might also affect the replicability of the results in applied contexts.

Another question that might be raised regards the compliance of participants with the given instruction. For instance, we assumed that the psychiatric inpatients responded honestly, while the students in the fake bad condition dissimulated their responses. However, some of the individuals in each of these groups might fail to attend the given instructions. Maybe some students failed to follow the faking instruction. Maybe some psychiatric patients were motivated to exaggerate their symptoms to get additional medical attention. We did not include formal manipulation check measures to exclude such participants from their assigned group.

Another potential limitation was given by the heterogeneity of the situations that simulators were allowed to choose from. For instance, underreporting in a personnel selection context might differ from the case found in a child custody examination context. In the first case, maybe the person adopts a strategy of responding that would highlight his or her "good qualities." In contrast, in the second case, the person might focus on "concealing problems." In this regard, the DS scale content seems to fit better for the detection of patterns of responses that highlight "unlikely virtues" than those which reveal "the denial of psychological shortcomings." The DF scale uses a reverse strategy by focusing on identifying a tendency to deny problems. Further research should explore specific contexts of under- and overreporting and whether different patterns of detection are found in each of such contexts.

In conclusion, the PCF validity scales seem to work appropriately to distinguish accurate personality profiles from distorted profiles, regardless of the direction of the distortion (overreporting, underreporting, or random reporting). However, future studies using more extensive and more ecological samples are required to replicate the obtained results and provide further insights into each validity scale's use in an applied context.

\section{References}

American Psychiatric Association. (2013). Diagnostic and statistical manual of mental disorders (5th ed.). Arlington, VA: Author

Baer, R. A., Wetter, M. W., Nichols, D. S., Greene, R., \& Berry, D. T. (1995). Sensitivity of MMPI-2 validity scales to underreporting of symptoms. Psychological Assessment, 7(4), 419-423.

Bagby, R. M., \& Sellbom, M. (2018). The validity and clinical utility of the personality inventory for DSM- 5 response inconsistency scale. Journal of Personality Assessment, 100(4), 398-405. doi: 10.1080/00223891.2017.1420659

Bass, C., \& Halligan, P. (2014). Factitious disorders and malingering: challenges for clinical assessment and management. The Lancet, 383(9926), 1422-1432. doi: 10.1016/ S0140-6736(13)62186-8

Butcher, J. N., Dahlstrom, W. G., Graham, J. R., Tellegen, A., \& Kaemmer, B. (1989). Manual for the administration and scoring of the MMPI-2. Minneapolis, MN: University of Minnesota.

Butcher, J. N., \& Han, K. (1995). Development of an MMPI-2 scale to assess the presentation of self in a superlative manner: The S Scale. In J. N. Butcher \& C. D. Spielberger (Eds.), Advances in personality assessment, Vol. 10 (p. 25-50). Lawrence Erlbaum Associates, Inc.

Chiracu, A. (2019). The role of resilience in the relationship between stressful life events and health in a Romanian sample. Romanian Journal of Applied Psychology, 21(2), 61-66. doi: 10.24913/rjap.21.2.04

Daubert, S. D., \& Metzler, A. E. (2000). The detection of fakebad and fake-good responding on the Millon Clinical Multiaxial Inventory III. Psychological Assessment, 12(4), 418-424. doi: 10.1037/1040-3590.12.4.418

Flaskerud, J. H. (2012). Cultural bias and Likert-type scales revisited. Issues in Mental Health Nursing, 33(2), 130132. doi: $10.3109 / 01612840.2011 .600510$

Hawes, S. W., \& Boccaccini, M. T. (2009). Detection of overreporting of psychopathology on the Personality Assessment Inventory: a meta-analytic review. Psychological Assessment, 21(1), 112-124. doi: $10.1037 / \mathrm{a} 0015036$

Kritzler, S., Krasko, J., \& Luhmann, M. (2020). Inside the happy personality: Personality states, situation experience, and state affect mediate the relation between personality and affect. Journal of Research in Personality, 85, 103923. doi: 10.1016/j.jrp.2020.103929

Krueger, R. F., Derringer, J., Markon, K. E., Watson, D., \& Skodol, A. E. (2013). The personality inventory for DSM5 (PID-5)-adult. American Psychiatric Association: Author.

Millon, T., Grossman, S., \& Millon, C. (2015). MCMI-IV. Bloomington, MN: NCS Pearson.

Morey, L. C. (2007). Personality assessment inventory (PAI): professional manual. PAR (Psychological Assessment Resources).

Nicholson, R. A., Mouton, G. J., Bagby, R. M., Buis, T., Peterson, S. A., \& Buigas, R. A. (1997). Utility of MMPI2 indicators of response distortion: Receiver operating characteristic analysis. Psychological Assessment, 9(4), 471-479. doi: 10.1037/1040-3590.9.4.471

Rogers, R., Sewell, K. W., Martin, M. A., \& Vitacco, M. J. (2003). Detection of feigned mental disorders: A metaanalysis of the MMPI-2 and malingering. Assessment, 10(2), 160-177. doi: 10.1177/1073191103010002007

Sava, F.A. (in press). Personality Clinical Form. The User's Manual. 
Sava, F.A. (2013). Psihologia validată stiintific. Ghid practic de cercetare științifică [Evidence-based psychology. A practical guide for psychological research]. Iași: Polirom

Schoenberg, M. R., Dorr, D., \& Morgan, C. D. (2003). The ability of the Millon Clinical Multiaxial Inventory--Third Edition to detect malingering. Psychological Assessment, 15(2), 198-204. doi: 10.1037/1040-3590.15.2.198

Sellbom, M., Dhillon, S., \& Bagby, R. M. (2018). Development and validation of an Overreporting Scale for the Personality Inventory for DSM-5 (PID5). Psychological assessment, 30(5), 582-593. doi: $10.1037 /$ pas0000507

StataCorp. (2017). Stata Statistical Software: Release 15. College Station, TX: StataCorp LLC.
Williams, M. M., Rogers, R., Sharf, A. J., \& Ross, C. A. (2019). Faking good: An investigation of social desirability and defensiveness in an inpatient sample with personality disorder traits. Journal of Personality Assessment, 101(3), $10.1080 / 00223891.2018 .1455691$

Youngstrom, E. A. (2014). A primer on receiver operating characteristic analysis and diagnostic efficiency statistics for pediatric psychology: we are ready to ROC. Journal of Pediatric Psychology, 39(2), 204-221. doi: 10.1093/jpepsy/jst062 